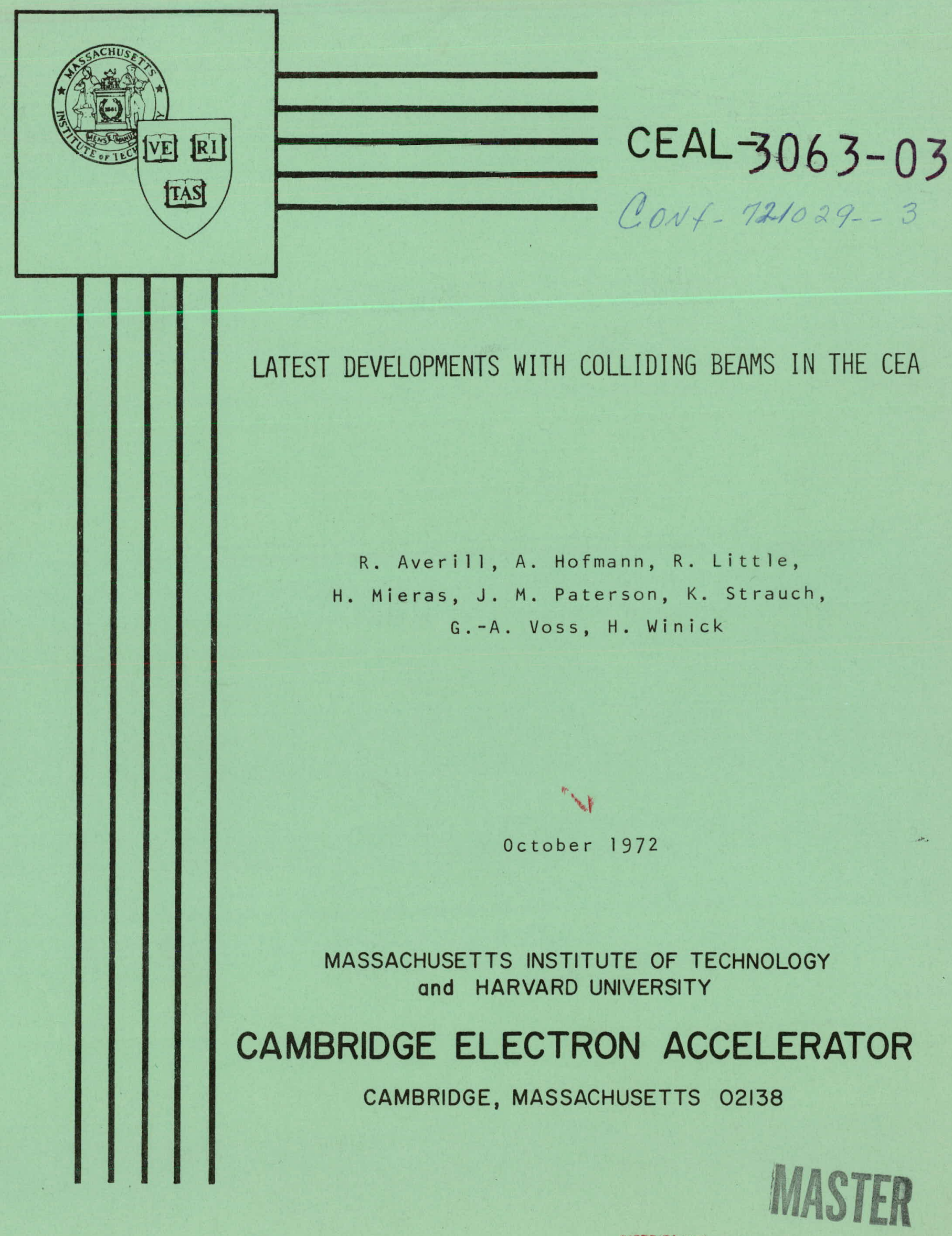




\section{DISCLAIMER}

This report was prepared as an account of work sponsored by an agency of the United States Government. Neither the United States Government nor any agency Thereof, nor any of their employees, makes any warranty, express or implied, or assumes any legal liability or responsibility for the accuracy, completeness, or usefulness of any information, apparatus, product, or process disclosed, or represents that its use would not infringe privately owned rights. Reference herein to any specific commercial product, process, or service by trade name, trademark, manufacturer, or otherwise does not necessarily constitute or imply its endorsement, recommendation, or favoring by the United States Government or any agency thereof. The views and opinions of authors expressed herein do not necessarily state or reflect those of the United States Government or any agency thereof. 


\section{DISCLAIMER}

Portions of this document may be illegible in electronic image products. Images are produced from the best available original document. 
The research work described in this report was performed under Contract AT $(30-1)-2076$ between

the U.S. Atomic Energy Commission and the President and Fellows of Harvard College. 


\title{
LATEST DEVELOPMENTS WITH COLLIDING BEAMS IN THE CEA*
}

R. Averlll, A. Hofmann, R. Little,

H. Mieras, J. M. Paterson, K. Strauch,

G. - A. Voss, H. Winick

\author{
Presented at the III National \\ Conference on Particle Accelerators \\ Moscow, USSR, October 2-4, 1972
}

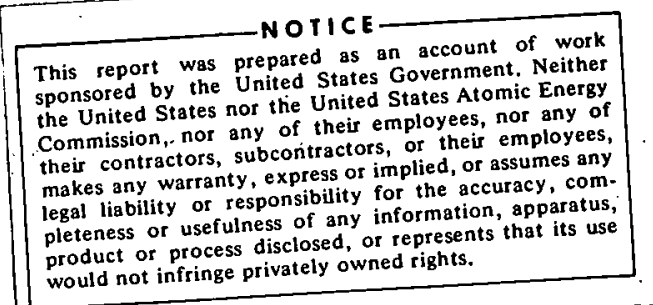

*Work supported by the U.S. Atomic Energy Commission under Contract No. AT $(11-1)-3063$. 
CEAL-3063-03

october 1972

\section{LATEST DEVELOPMENTS WITH COLLIDING BEAMS IN THE CEA}

The design of the CEA bypass, which makes it possible to use the 6-GeV electron synchrotron as a colliding beam facility, has been previously described.1,2,3 Status reports of the work on the bypass were given at the 7 th and 8 th 1 nternational Conferences on High Energy Accelerators at Yerevan (1969), and Geneva (1971).4,5 Since May of 1972, the CEA bypass has been in use for a colliding beam experiment with 2-GeV electron and positron beams. This report describes the present performance of the CEA colliding beam facility, its limitations, and some aspects of the experiment presently underway.

Positron injection into the CEA and subsequent accumulation is done in a multicycle injection mode where positrons of $140 \mathrm{MeV}$ from a linear accelerator are injected in a single turn off-axis into the synchrotron. 6 After acceleration to 2 GeV, and deceleration to $14.0 \mathrm{MeV}$, radiation damping has decreased the positron beam size and new additional injection is possible. At an injection rate of $60 \mathrm{~Hz}$, a positron fill-rate of $0.4 \mathrm{~mA}$ per second has been achieved. The lifetime of the stored beam in this cycling mode is 400 seconds, 1 imited by gas scattering. The positron current which can be accumulated is limited by beam instabilities; after elimination of betatron instabilities of the head-tail type, with a system of distributed sextupole magnets, positron currents are presently limited to $25 \mathrm{~mA}$ *

\footnotetext{
"All currents referred to in this report are "peak currents", i.e. instantaneous currents averaged over one $r f$ wavelength. Since the synchrotron is filled to the extent of only $1 / 3$ of the circumference, corresponding to 120 bunches in each beam (the $r f$ harmonic number is 360 ), the average circulating current is $1 / 3$ of the quoted "peak currents".
} 
by instabilities in the phase oscillation mode. It has been suspected for some time that this instability is a single-bunch phenomenon and that bunch-bunch interaction is not a significant contributor. This was confirmed when we succeeded in accumulating current in one bunch only. It was found that the instabllity ehreshold current per bunch was independent of the number of bunches in the $r i n g$ and that an unstable single bunch displays the same coherent motion of the center of charge as is observed with 120 bunches (Fig. 1). A model to explain such single-bunch instabilities would have to assume radially dependent azimuthal electric fields produced by a bunch and acting back on it. Attempts to calculate the fields excited by the beam inside the ceramic vacuum chamber in the CEA have not yet been successful due to the great complexity of the system. However the existence of very strong beam-induced fields can be observed by measuring the betatron frequency for coherent beam motion as a function of beam current (Fig. 2). It is found that the change of vertical betatron frequency for coherent beam oscillations is directly proportional to the current in a single bunch, independent of the number of bunches. The magnitude of this effect is close to that which one would expect if the image currents in the ceramic chamber wall would produce only electric fields and no compensating magnetic fields. Although the fields which produce.this change in nuvalue have the wrong direction and not necessarily the right radial dependence to produce phase instabilities, they are by several orders of magnitude stronger than those necessary to produce those phase instabilities, and the existence of some small components with the right direction and radial dependence is easily imaginable.

Several ways to improve the current limits as given by phase instabilities have been explored. To increase the Landau damping due to the nonlinearity of the accelerating $r f$ waveform, we operate at the lowest possible rf voltage. A special accelerating cavity operating on the third harmonic (1428 $\mathrm{MHz}$ ) of the main $r f$ system has been installed to increase further the nonlinearity of the $r f$ waveform. Lack of an adequate power supply for the operation of this cavity has limited the 
improvement to less than $30 \%$. A cavity operating on a different harmonic number (361) eliminates bunch-bunch interaction but since the instability is dominantly a single-bunch phenomenon, the effect of this cavity is not very significant.

After the synchrotron ring has been filled with positrons, the minimum energy of the synchrotron is raised to $260 \mathrm{MeV}$, electrostatic fields are turned on to vertically separate electron and positron beams, and multicycle electron injection proceeds. Due to the higher minimum energy and consequentiy smaller adiabatic antidamping during the electron injection cycle, the electron beam size at the minimum energy is considerably smaller than the positron beam size during positron injection and therefor more aperture is available for beam growth due to instabilities. This, together with the more plentiful electron currents available, makes it possible to accumulate up to 150 $m A$ of electrons in the synchrotron. But as soon as the electron current exceed values of 30 to $70 \mathrm{~mA}$, the lifetime of the stored positron current is drastically reduced through longrange beam-beam interaction. This effect of the electrons on the positrons not only changes the betatron frequencies for the positron beam such that the operating point for positrons is moved onto resonances, but it also creates a number of new nonlinear resonance stopbands which then cause positron beam loss. The smallest beam-beam separation in the cycling mode is approximately $2 \mathrm{~mm}$ at $E_{\max }$, the maximum energy, and $6 \mathrm{~mm}$ at Emin, the minimum energy. It is limited at Emax by the high voltage capabilities of the electrostatic plate system, at $E_{m i n}$ by the vertical aperture requirements at injection. The present electrostatic plate system unfortunately provides beambeam separations which vary by a factor of 2 along the circumference. This is the reason why the smallest separation at Emin cannot be increased much beyond the quoted $6 \mathrm{~mm}$. A new electrostatic plate separation system, which not only provides twice the beam-beam separation at $E_{m a x}$ but also does this in a more uniform way (and thereby allows an increase of the smallest beam-beam separation at $E_{m i n}$ by a factor of 2 ), is being installed. With this new system we expect a considerable improvement in the beam-beam interaction limit. 
After the synchrotron ring has been filled with electron and positron currents, the ac component of the synchrotron magnet is turned off and the dc component is adjusted for the energy at which the colliding beam experiment is to be done. The beams are then switched into the bypass. The switching is typically done with losses of 0 to $10 \%$ Llfetimes of stored beams going through the bypass are between one and two hours. corresponding to an air equivalent average pressure of $10^{-8}$. Torr, and are still improving with time due to the gradual improvement of thc vacuum.

The bypass is characterized by a low-beta interaction. point where electron and positron beams cross each other at an angle of typically $2.4 \mathrm{mrad}$. Present values of the amplitude function at the interaction point are $\beta_{h}=4.6 \mathrm{~cm}$ and ${ }^{2} v=28$ $\mathrm{cm}$. The momentum vector at this point is zero. At 2 GeV the beam size at the interaction point has been found to be $0.13 \mathrm{~mm}$ wide and $0.10 \mathrm{~mm}$ high. The beam width is in good agreement with the theoretical width, as given by quantum fluctuations. The beam height on the other hand is mostly determined by cross-coupling effects. A large portion of this cross coupling is produced in the distributed sextupole system where beams, because of their vertical separation, cannot go through the center of the sextupoles. These effects can in principle be corrected with twisted electrostatic quadrupoles.

A large number of nonlinear sum and difference resonances up to 6 th order, together with satellites of the main resonances, restrict the range of betatron frequencies over which one finds stable operating conditions. Quadrupole currents in the bypass have to be controlled to an accuracy of $10^{-3}$ and the energy of the synchrotron has to be stable to better than $10^{-4}$.

In a typical run for an experiment the beams are kept for 20 minutes in the bypass. Refilling the ring with positrons and electrons and switching the beams back into the bypass takes between 5 and 10 minutes. In this way the time average luminosity is optimized and is equal to about half of the peak luminosity. The luminosity is measured through double bremsstrahlung (Fig. 3a). The maximum luminosity at 2 GeV has been 
measured to be $3.0 \times 10^{28} \mathrm{~cm}^{-2} \mathrm{sec}^{-1}$. This luminosity was achieved with a total average circulating current of $7.5 \mathrm{~mA}$, about equally divided into, $3.75-\mathrm{mA}$ average current in each beam. The peak current in each beam had values up to $20 \mathrm{~mA}$, but because of unavoidably nonuniform filling of the beam sausages, the current averaged over the sausages (approximately $1 / 3$ of the circumference) was only $11 \mathrm{~mA}$. With these small currents background counts in the luminosity measurement (Fig. $3 \mathrm{~b}$ ) and in the detector surrounding the interaction region are very small. The present bypass on-line detector (BOLD) has been built by a Harvard-MIT-CEA group under R. Little. The detector (Fig. 4) covers a solid angle of $2 \pi$ (or $50 \%$ of $4 \pi$ ) around the interaction point with wire spark chambers, scintillators and absorbers, and operates on-line with the Harvard IBM 370/145 computer. Since the interaction volume is small in every dimension as compared to the volume covered by the detector, a good discrimination against gas background, machine background, and cosmic-ray background events, is given by the computerized reconstruction of tracks to their origin. Figure 5 shows the points of origin for 100 wide-angle electron-electron scattering events. The total l/e length of the interaction volume of $3.5 \mathrm{~cm}$ is in good agreement with the $\sqrt{2}$ times longer theoretical $1 / \mathrm{e}$ bunch length of $5 \mathrm{~cm}$. The total $1 / \mathrm{e}$ width of $0.3 \mathrm{~cm}$ of the interaction volume is given by the resolution of the spark chambers extrapolated to the interaction point. The first experimental results, among them the surprisingly large production cross section for multi-hadronic events, have been described at the 16 th International Conference on High Energy Physics, NAL, Batavia, Illinois, U.S.A., September 1972. Two computer. replays of events are shown in Fig. 6.

At the present time the CEA colliding beam facility is shut down to facilitate some major improvements. The abovementioned installation of a new electrostatic plate separation system is expected to greatly reduce beam-beam interaction and. allow larger currents. With the additional installation of two more twisted electrostatic quadrupoles (two have already been installed) we expect to reduce the vertical beam size by at least a factor of two. We hope that these two changes will 
increase the obtainable luminosity into the $10^{29} \mathrm{~cm}^{-2} \mathrm{sec}^{-1}$ range. Although the bypass has already operated at energies of $2.5 \mathrm{GeV}$ in each beam, it is believed that the quality of the present quadrupoles close to the interaction region is insufficient to increase the beam energy further. For this reason a new set of quadrupoles with greatly improved linearity is being installed. With this we expect to extend the range of energies in each beam to 3 GeV and perhaps higher. 


\section{References}

1 A. Hofmann et al., "The Colliding Beam Project at the CEA", Proceedings $6 \mathrm{th}$ International Conference on High Energy. Accelerators, Cambridge, Mass., U.S.A., 1967, CEAL-2000, P.112.

2 H. Mieras et al., "A Design of the CEA Colliding Beam Bypass", Proceedings $6 \mathrm{th}$ International Conference on High Energy ACcelerators, CEAL-2000, p. 119.

3 A. Hofmann et al., "Design and Performance of the Damping System for Beam Storage in the CEA", Proceedings 6 th International Conference on High Energy Accelerators, CEAL-2000, p. 123 .

4 G.-A. Voss, "Status of the CEA Bypass for Colliding Eeams", Proceedings 7 th International Conference on High Energy Accelerators, Yerevan, Armenia, U.S.S.R., 1969, Vol. 11, p. 48.

5 R. Averill et al., "Colliding Electron and Positron Beams in the CEA Bypass", Proceedings 8 th International Conference on High Energy Accelerators, Geneva, Switzerland, 1971, p. 140.

6 K. Robinson et al, "A Proposed System for Multicycle Injection of Positrons and Electrons into the 6-GeV Cambridge Electron Áccelerator", IEEE Transactions on Nuclear Science, Vol. NS-14, No. 3, p. 670, June 1967. 
List of 111ustrations

Figure 1

Figure 2

Figure 4

Figure 5

Figure 6
Frequency analysis of oscillations of a single bunch.

Vertical betatron frequency as a function of the peak circulating current.

Figure $3 a$ and $3 b$ Luminosity measurement using double brewsstrahlung.

View of the interaction region with part of the detector.

Plot of the origins of 100 large-angle $e^{+} e^{-}$ events.

Two examples of events detected with the BOLD detector.

$-8-$ 


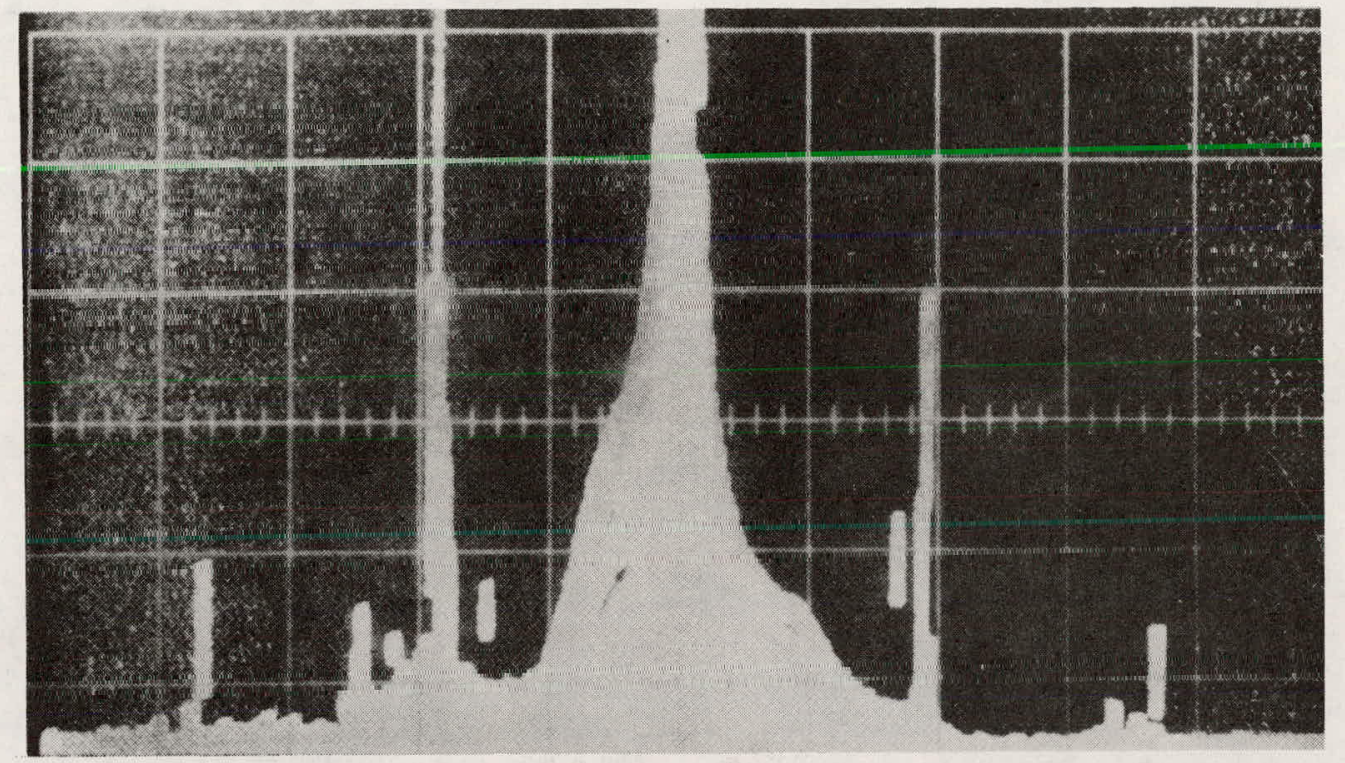

Fig. 1. Frequency analysis of horizontal displacement signal of a single bunch. Horizontal scale is frequency at $50 \mathrm{KHz} / \mathrm{cm}$. The ordinate represents amplitude, with arbitrary scale. The large central peak is at a multiple of the circumferential frequency of $1.3 \mathrm{MHz}$, indicating an orbit distortion at the location of the monitor. The first main sidebands at central frequency $\pm 100 \mathrm{KHz}$ are due to a phase instability which causes coherent oscillation of the center of charge. 


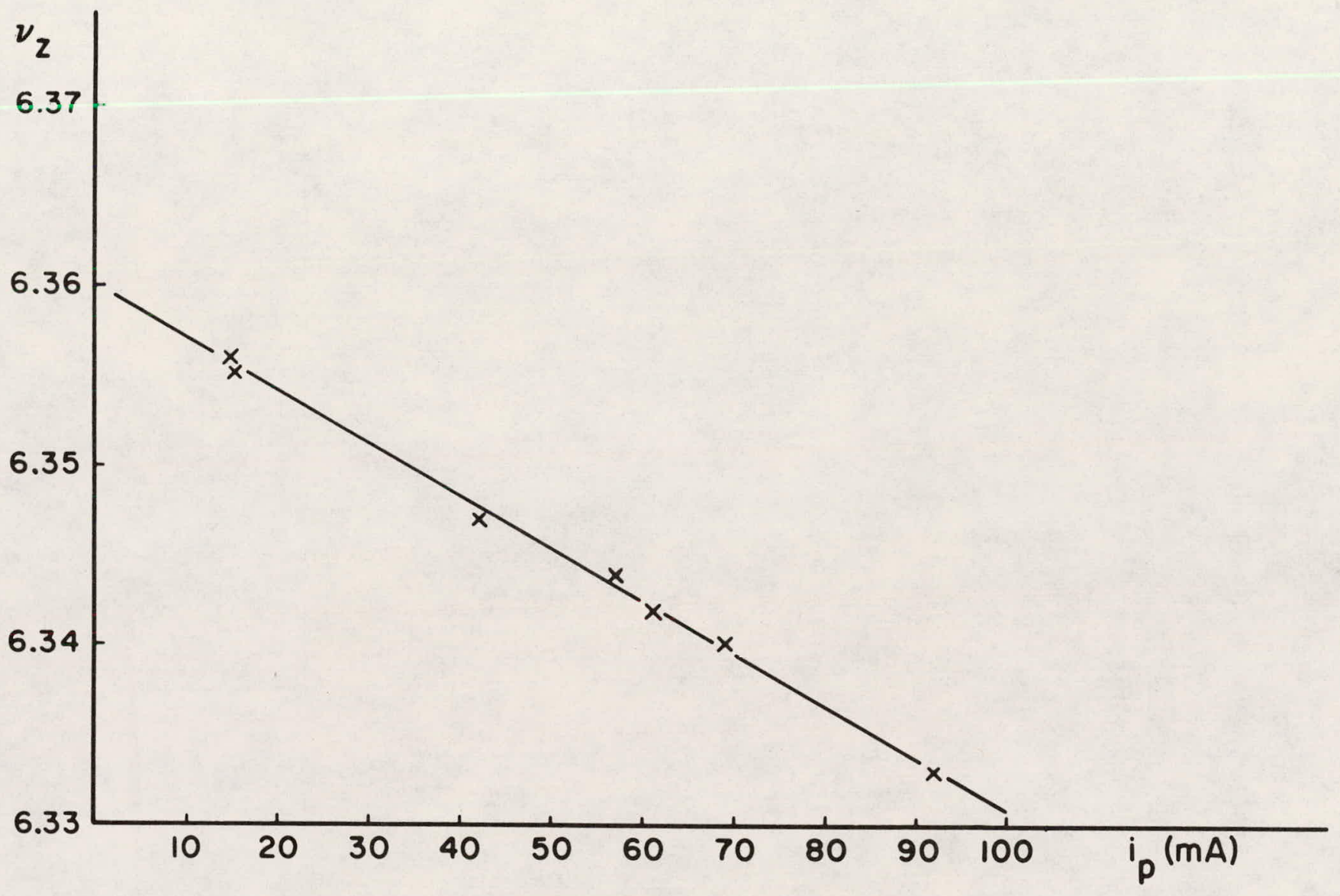

Fig. 2. Vertical betatron frequency $\nu_{z}$ as function of the peak circulating current ip. (ip is the current averaged over one rf wavelength.) 


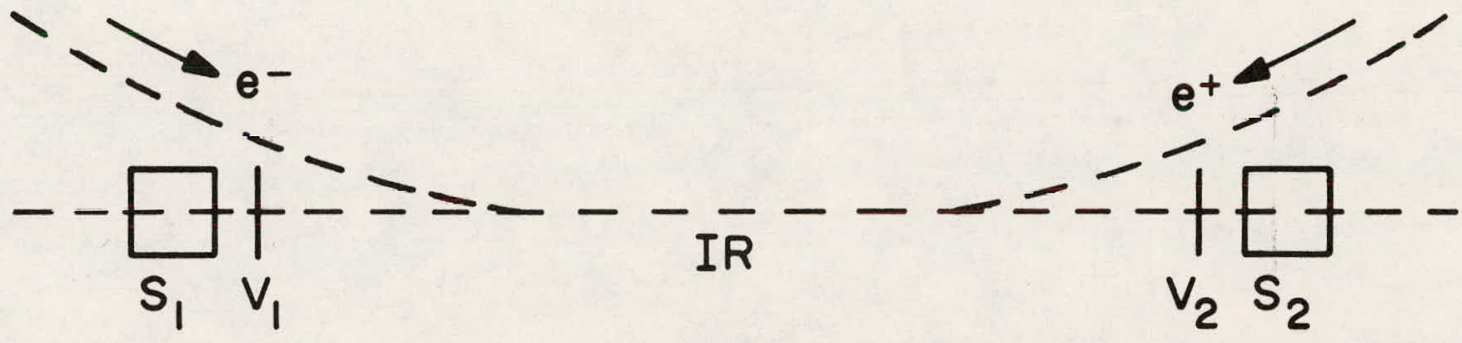

Fig. 3a. Schematic of arrangement for measuring luminosity by using double bremsstrahlung. IR = interaction region, $V=$ veto counter, $S=$ shower counter.

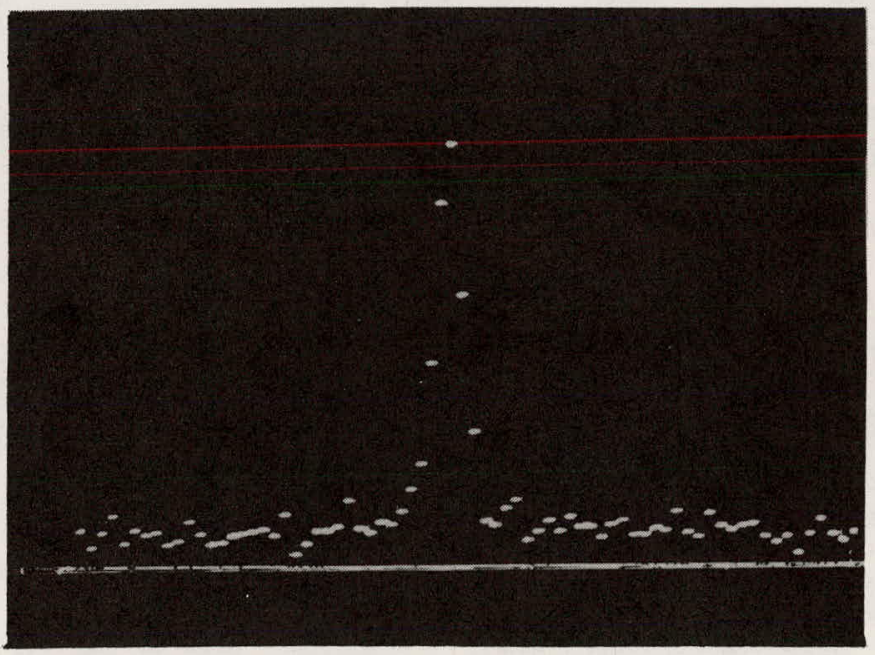

Fig. 3b. Scope display of a luminosity measurement using double bremsstrahlung. Horizontal scale is proportional to time difference between a count in $S_{1}$ and the next, delayed by about 50 nsec, count in $\mathrm{S}_{2}$. Ordinate is proportional to number of counts falling into a channel. (Channel width approximately 1.5 nsec.) 


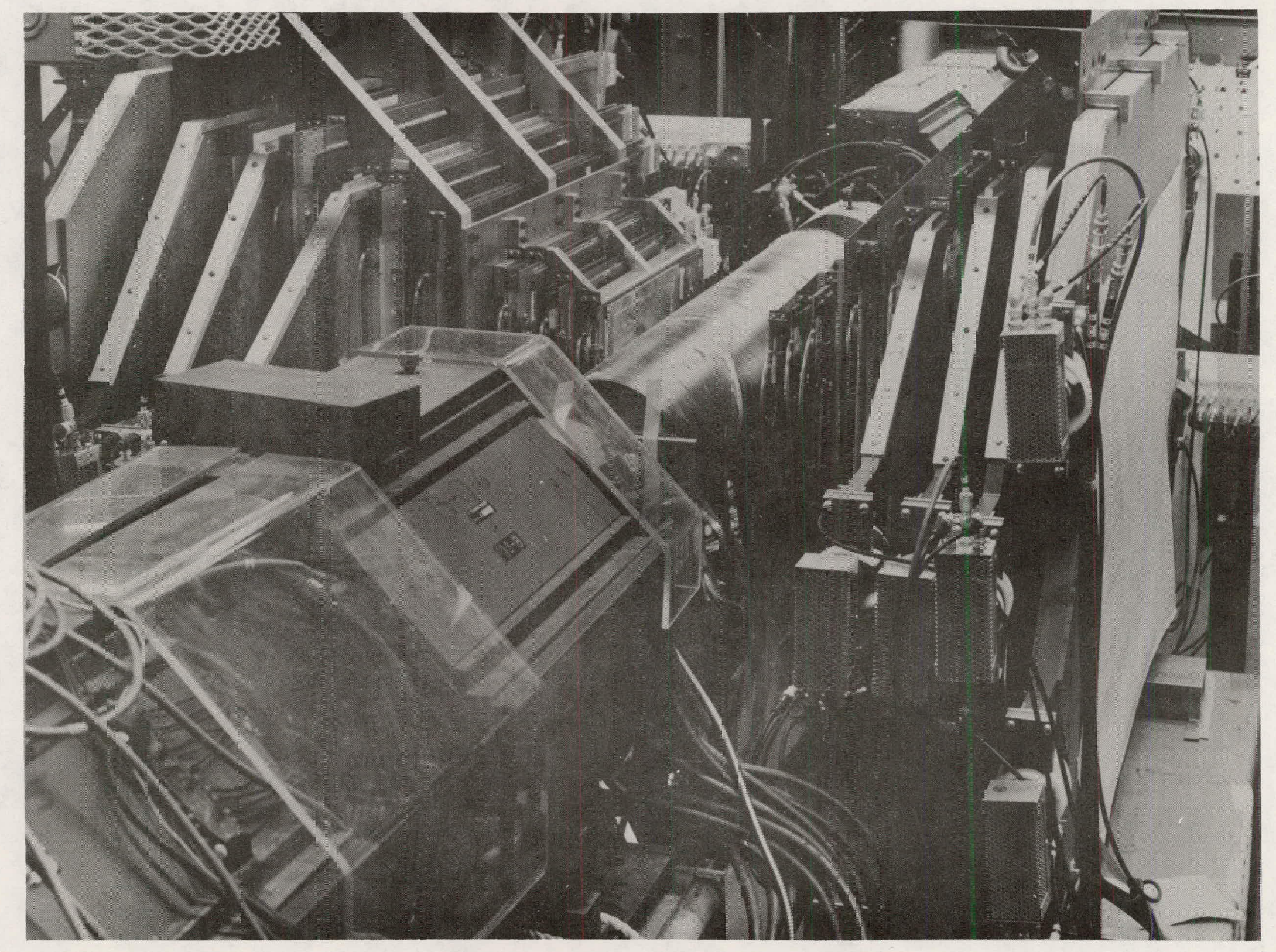

Fig. 4. View 0 the interaction region seampipe and two quadrants of the BOLD cetector. (The top ant bottom quadrant lad been taken out when this picture was taken.) The length of the straight section between the two quadrupoles next to the interaction region is 2.95 meters. 


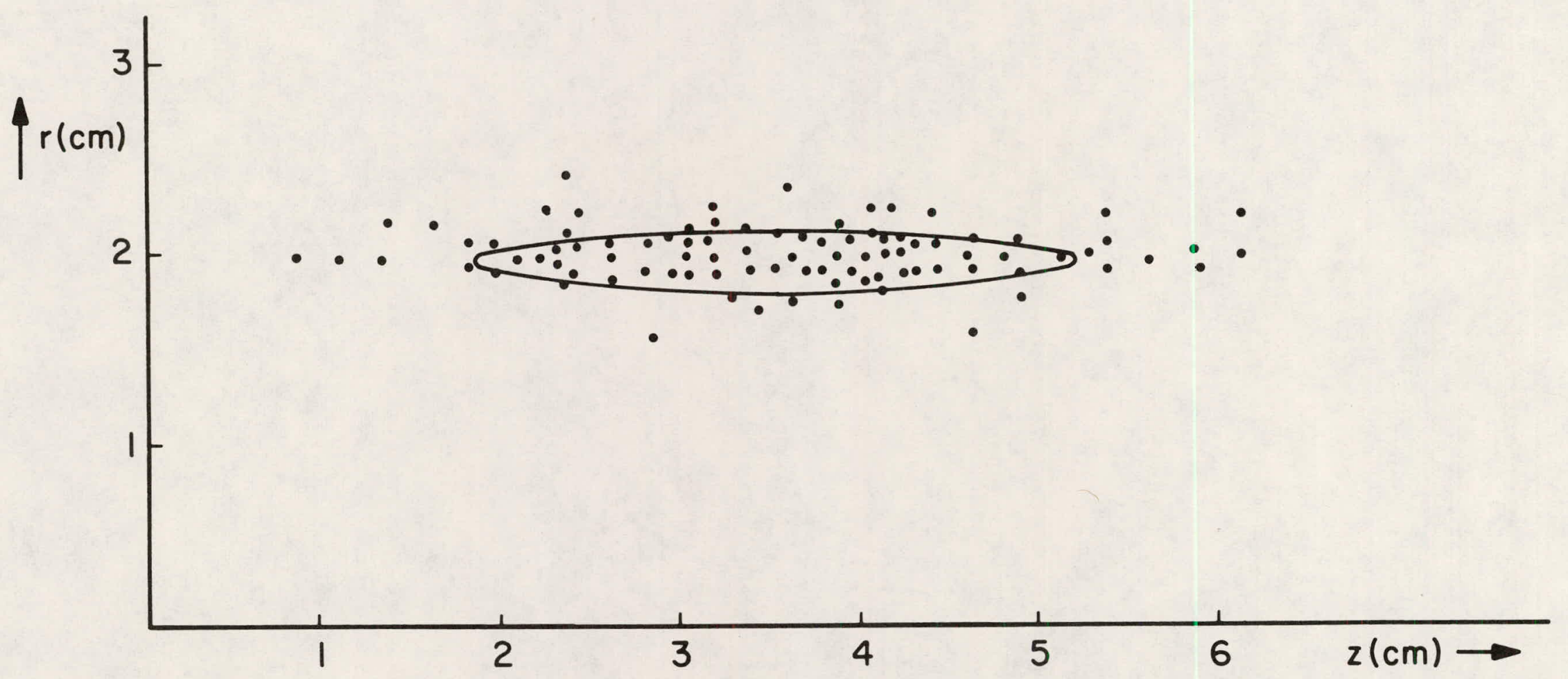

Fig. 5. Points of origin of 100 vertical large angle $e^{+} e^{-}$scattering events in a horizontal plane ( $z$ is the direction of the beams). The plotted l/e contour indicates a length of the interaction volume of $3.5 \mathrm{~cm}$, determined by the bunch length of $5 \mathrm{~cm}$. The horizontal width of $0.3 \mathrm{~cm}$ is determined by the spatial resolution of the spark chambers, extrapolated to the interaction point. 
a)

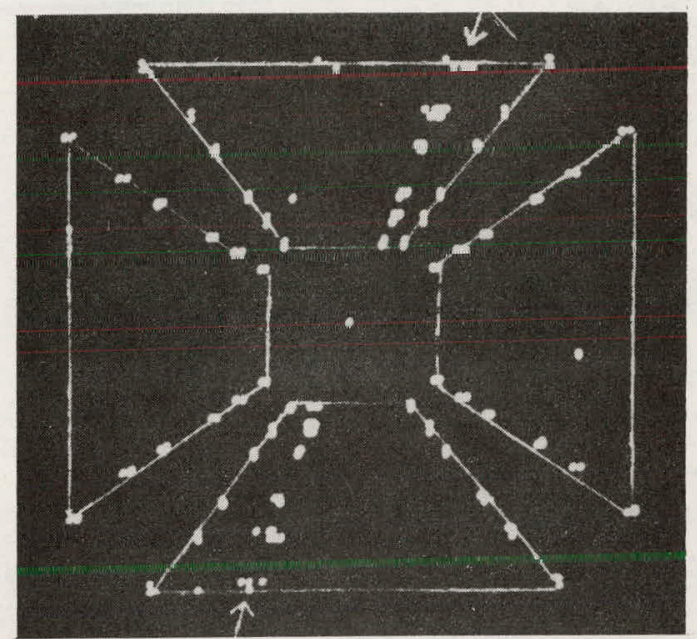

b)

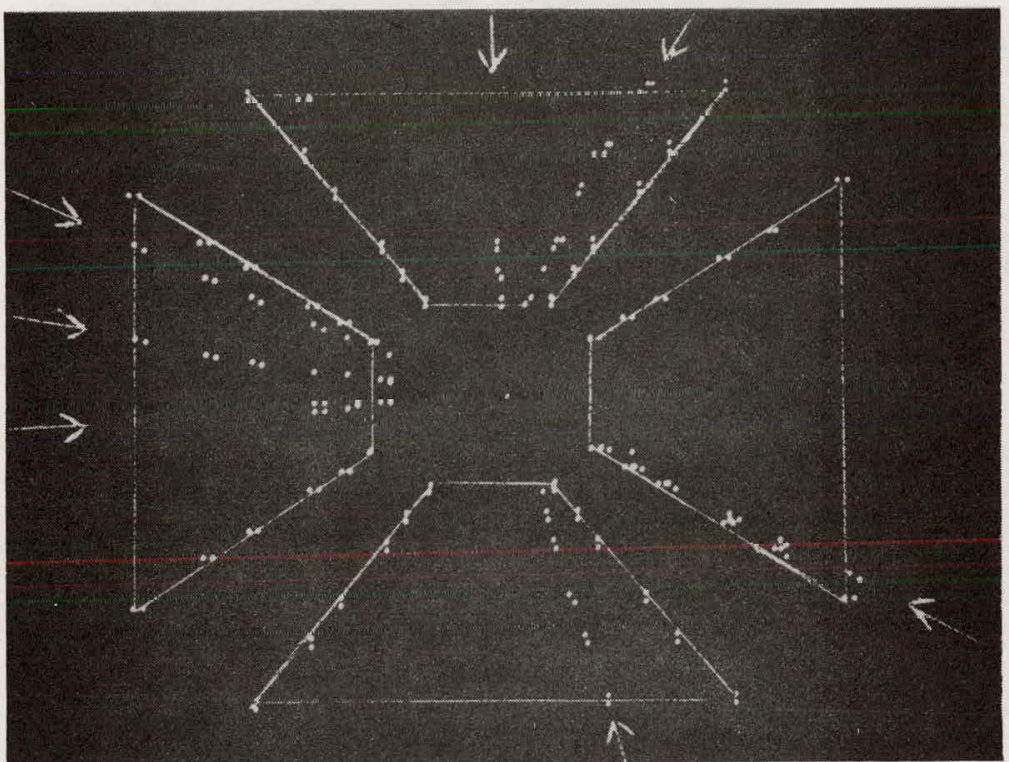

Fig. 6. Two events detected in BOLD

a) A typical large angle $e^{-} e^{+}$scattering event. Note the development of the electromagnetic showers in planes 4, 5 , and 6 (planes counted from the interaction region) produced by lead absorbers between planes 3 and 4 , 4 and 5 , and 5 and 6 .

b) An example of one of the interesting multi-hadronic events.

Both views are in the direction along the beam runs. The arrows indicate the particle tracks. 
LEGAL NOTICE

This report was prepared as an account of U. S. Government sponsored work under the auspices of the U. S. Atomic Energy Commission. Neither the Inited Etates, luur the commission, nor any person acting on behalf of the Commission:

A. Makes any warranty or representation, express or implied, with respect to the accuracy, completeness, or usefulness of the information contained in this report, or that the use of any information, apparatus, method, or process disclosed in this report may not infringe privately owned rights; or

B. Assumes any liabilities with respect to the use of, or for damages resulting from the use of any information, apparatus, method, or process disclosed in this report.

As used in the above, "person acting on behalf of the Commission" includes any employee or contractor of the Commission to the extent that such employee or contractor prepares, handles or distributes, or provides access to any information pursuant to his employment or contract with the Commission. 